\title{
Hallazgos monetales en la Alcazaba de Talavera de la Reina (Toledo). Campañas 2007-2011*
}

\section{Coin finds in the Alcazaba of Talavera de la Reina (Toledo). 2007-2011 Archaeological campaigns}

\author{
Alberto Moraleda Olivares - Sergio Sánchez Sanz \\ Sergio de La Llave Muñoz**
}

\begin{abstract}
RESUMEN
El presente estudio numismático aborda los hallazgos monetales recuperados durante las campañas de excavación llevadas a cabo entre los años 2007 y 2011. Para contextualizar los hallazgos recurrimos a la información histórica y arqueológica. Como resultado, se presenta aquí una aproximación a la circulación monetaria en el espacio de la Alcazaba de Talavera.
\end{abstract}

PALABRAS CLAVE

numismática, monedas, circulación monetaria, Talavera de la Reina, alcazaba.

\begin{abstract}
This study focuses on the coins discovered during the archaeological campaigns carried out between 2007 and 2011. Historical and archaeological information has been used in order to classify the finds. We will present our findings, as a first approach to coin circulation in the Alcazaba of Talavera de la Reina (Toledo).
\end{abstract}

KEY WORDS

numismatics, coins, monetary circulation, Talavera de la Reina, alcazaba.

\section{INTRODUCCIÓN}

Damos a conocer un conjunto de monedas halladas durante las campañas arqueológicas desarrolladas en la Alcazaba de Talavera de la Reina entre los años 2007-2011, cuyos trabajos fueron dirigidos por Alberto Moraleda y Sergio

\footnotetext{
* Fecha de recepción del artículo: 2012-11-29. Fecha de aceptación del trabajo: 2013-2-1.

" Alberto Moraleda: Arqueólogo. Centro Asociado a la UNED de Talavera de la Reina. C.e.: albertomoraleda@yahoo.es; Sergio Sánchez Sanz: Arqueólogo. C.e.: 2909al@terra.es; Sergio de la Llave Muñoz: Arqueólogo. C.e.: sergiodelallave@ outlook.com.
} 
Sánchez. Con este estudio se pretende aportar nuevos datos sobre la circulación monetaria en época antigua, medieval y moderna en Talavera de la Reina, ya que se ha obtenido material suficiente asociado a niveles arqueológicos que nos da información sobre los usos del espacio de la Alcazaba.

El solar actual, donde fue ubicada la Alcazaba musulmana de Talabîra, coincide con el espacio conocido popularmente como «Huerto de San Agustín». Localizado en el vértice suroriental del primer recinto amurallado, engloba un área de unos 110 $\mathrm{m}$ de longitud por 90 de ancho, ocupando una superficie de unos $8650 \mathrm{~m}^{2}$, extendiéndose desde la torre . $^{\circ} \mathbf{1}$ hasta la torre . $^{\circ} \mathbf{6}$, ambas inclusive (Fig. 1).

El interior del «Huerto de San Agustín» estuvo activo como huerta hasta el verano de 1988. Presentaba un aspecto típico de huerta, con frutales, cultivos, así como infraestructuras relacionadas con dicha actividad (alberca, canalizaciones, etc.), aprovechando como casa de labor una edificación del siglo XVII de dos plantas realizada con el tradicional aparejo toledano con cobertizo exterior, construida por los monjes del Convento de Agustinos Recoletos y que se unía a él mediante cobertizo volado sobre la calle Pescaderías.

La primera intervención arqueológica se efectuó en el año 1989 dirigida por Villa González (1992: 371-392). El objetivo de la misma era la documentación de posibles restos arqueológicos que pudieran verse afectados por un proyecto urbanístico que el propietario del solar del citado huerto pretendía llevar a cabo. Esto permitió constatar la existencia de restos arquitectónicos presumiblemente pertenecientes al periodo califal y que corresponderían con elementos de la Alcazaba islámica, ocupando un asentamiento romano anterior del que también se documentaron restos estructurales.

Será en el año 2000 cuando comienza a gestionarse y a tomar realidad el primer proyecto de investigación arqueológica de Talavera de la Reina, con el Proyecto de investigación del primer recinto amurallado de Talavera de la Reina, Sector Entretorres (Moraleda, Martínez y Sánchez, 1999-2000: 54-70; Moraleda, Sánchez y Martínez, 2004: 409-421 y Martínez, Moraleda y Sánchez, 2005: 117-154). A raíz de este inicio, se forma un equipo de investigación de las murallas de Talavera de la Reina, que ha venido estudiando el recinto amurallado hasta la actualidad. Esta investigación ha abarcado varios sectores: Entretorres, Charcón (Moraleda et al., 2001, 2005, 2006 y 2007) y Alcazaba (Moraleda, 2001; Moraleda y Maquedano, 2002-2003; Moraleda et al., 2006-2007 y Moraleda y Sánchez, 2009 y 2010). Asimismo, y dentro de este proceso fue redactado el Plan director de las Murallas de Talavera de la Reina.

En 2009 el Ayuntamiento de Talavera de la Reina, pone en marcha una intervención global de rehabilitación de los paramentos de la muralla de la Alcazaba, sectores Ronda del Cañillo y calle Carnicerías. Esta intervención fue desarrollada en paralelo a una excavación en área del interior del recinto de la Alcazaba, la cual fue dividida en varios sectores con una extensión media de $400 / 450 \mathrm{~m}^{2}$ por área, resultando un total de 9 áreas de intervención. De este modo, desde septiembre de 2009 hasta agosto de 2010, con una ampliación posterior de los meses de octubre a diciembre, fueron exca- 
vadas las cuatro primeras áreas que se encuentran junto al intradós del lienzo de la muralla del sector Carnicerías (Moraleda y Sánchez, 2009 y 2010).

En total fue extraído un volumen total de $8.000 \mathrm{~m}^{3}$ de tierra, lo que ha permitido documentar la evolución histórica del yacimiento, donde se han constatado restos de diversa índole y cronología, que van desde época tardoantigua hasta la actualidad.

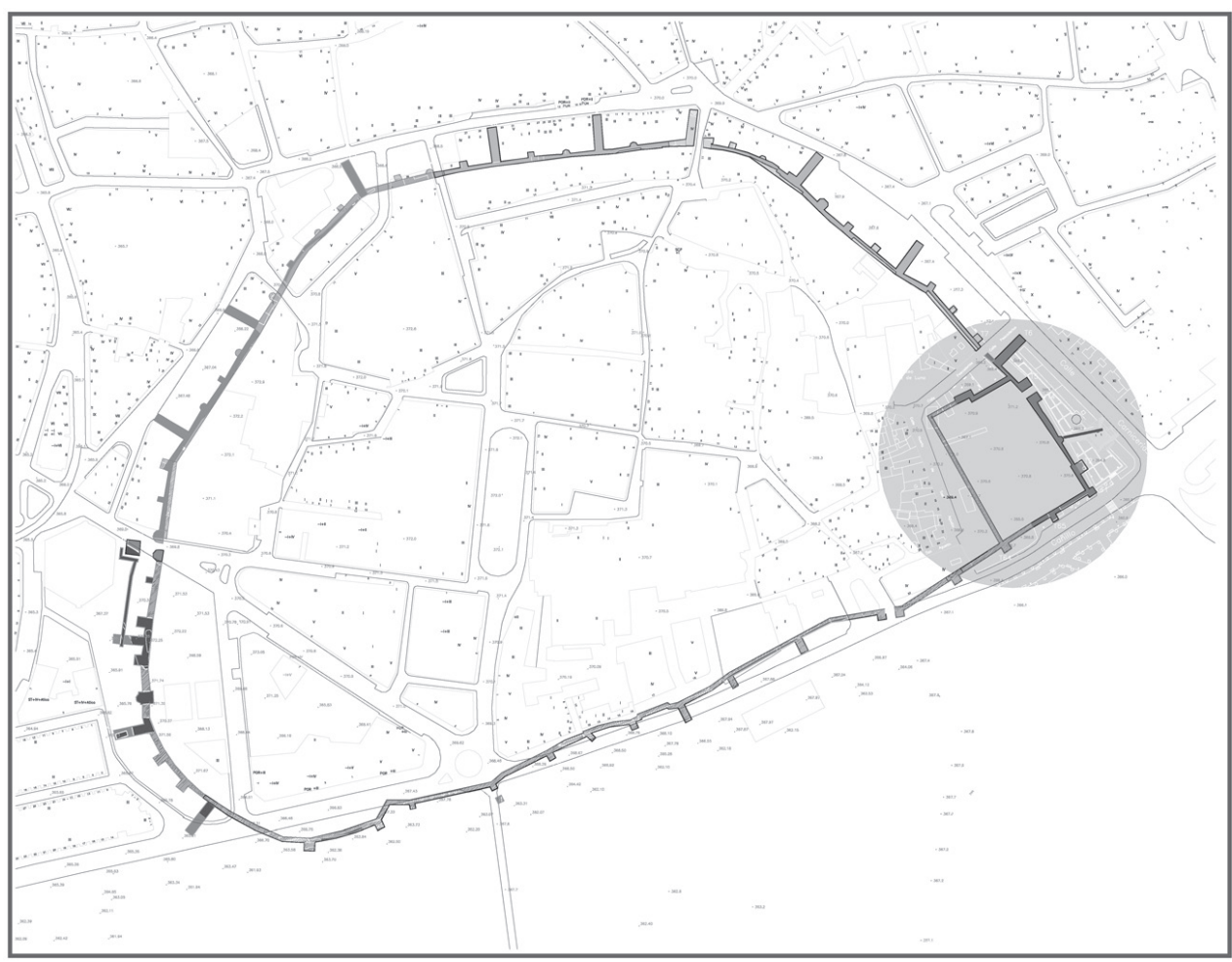

Fig. 1. Ubicación de la Alcazaba en el primer recinto amurallado (Moraleda y Sánchez).

\section{CONTEXTO HISTÓRICO}

A pesar de que la historiografía moderna plantea la posibilidad de la existencia de un recinto amurallado que delimitase el núcleo urbano de Caesarobriga-Elbora o Talabira a lo largo de la tardoantigüedad y el emirato omeya, en el estado actual de la investigación no será hasta el momento califal andalusí (siglo X) cuando tengamos claras evidencias de la construcción de una muralla. 
La Alcazaba fue la residencia del gobernador omeya que durante la segunda mitad del siglo X se ocupó de velar por los intereses que la dinastía cordobesa tenía en la zona de la Meseta, con especial atención en la «vigilancia» de la medina levantisca de Toledo. Ahmad al-Razi (Levi-Provenzal, 1953: 82) afirma que en el año 325 H. (936/7 d.C.) el califa Abd al-Rahman III ordena la construcción de una Alcazaba para residencia del gobernador, y la separación entre la villa y el exterior, así como se procedería a cerrar el resto del recinto urbano por medio de una muralla levantada con sillares cuadrangulares, dispuestos con cierta regularidad a soga y tizón, reaprovechando materiales de antiguos edificios tardoantiguos. Su reforma o construcción debió finalizar en tiempos de Al-Hakam II, sucesor de Abd al-Rahman III, en el año 357 H. (967/8 d.C.) tal y como lo atestigua una inscripción que se encontraba empotrada en la torre 1, situada en el sector sureste de la Alcazaba (Canto y Rodríguez, 2006: 57-66).

En 1083, tras la caída de Talavera en manos de Alfonso VI, la antigua Alcazaba pasó a ser el nuevo Alcázar Cristiano, siendo la residencia del alcaide castellano, quien se vio obligado a abandonarlo tras el asedio y toma momentánea de la misma por parte de los almorávides a comienzos del siglo XI (año 1109/1110). Con la definitiva conquista de Alfonso VII, ya en la segunda década del siglo XII, se ratificaría el papel señorial de la primitiva alcazaba que se mantendrá hasta el año 1371, año en que la villa pasó a posesión del arzobispo de Toledo D. Gómez Manrique. A partir de ese momento, se convierte en palacio arzobispal, categoría que mantendrá hasta el año 1571. A comienzos del siglo XVI, el edificio entra en un proceso de ruina y abandono hasta su adquisición por parte de la comunidad de agustinos Recoletos, siendo entonces cuando se comience a utilizar como huerto lo largo del siglo XVI, uso que mantendrá esa congregación hasta las últimas décadas del siglo XVIII. A lo largo del siglo XIX y XX el «Huerto de San Agustín» ha mantenido la misma función hasta su adquisición por parte del ayuntamiento de Talavera de la Reina en el año 1999. Momento a partir del cual comenzarán a realizarse trabajos encaminados a la investigación arqueológica.

\section{HALLAZGOS NUMISMÁTICOS}

A continuación, se procede a contextualizar dentro del proceso de excavación los materiales recuperados en cada una de las áreas en las que se ha actuado.

\section{Área 1}

Las monedas halladas en esta zona $\left(\mathbf{n} .^{\circ} 6\right.$ y $\left.\mathbf{1 8}\right)$ fueron halladas en la U.E. 1023 , la $\mathbf{n} .^{\circ} \mathbf{6}$ de época romana (Teodosio I, s. IV), y la $\mathbf{n} .^{\circ} 18$ de época medieval (felús sin identificar, ss. X-XIV). Esta unidad se encuentra ubicada en el sector Noroeste del área de intervención, correspondiéndose con un estrato deposicional originado por la actividad de relleno de este espacio en un momento de abandono de todo este recin- 
to, momento en el que las gentes de la ciudad utilizaron dicho espacio como vertedero. Fruto de esta actividad es la causa por la cual nos encontramos dos elementos numismáticos de diversa cronología. Tal y como se ha mencionado, esta unidad estaría cubriendo los restos estructurales de la última fase del Alcázar Cristiano.

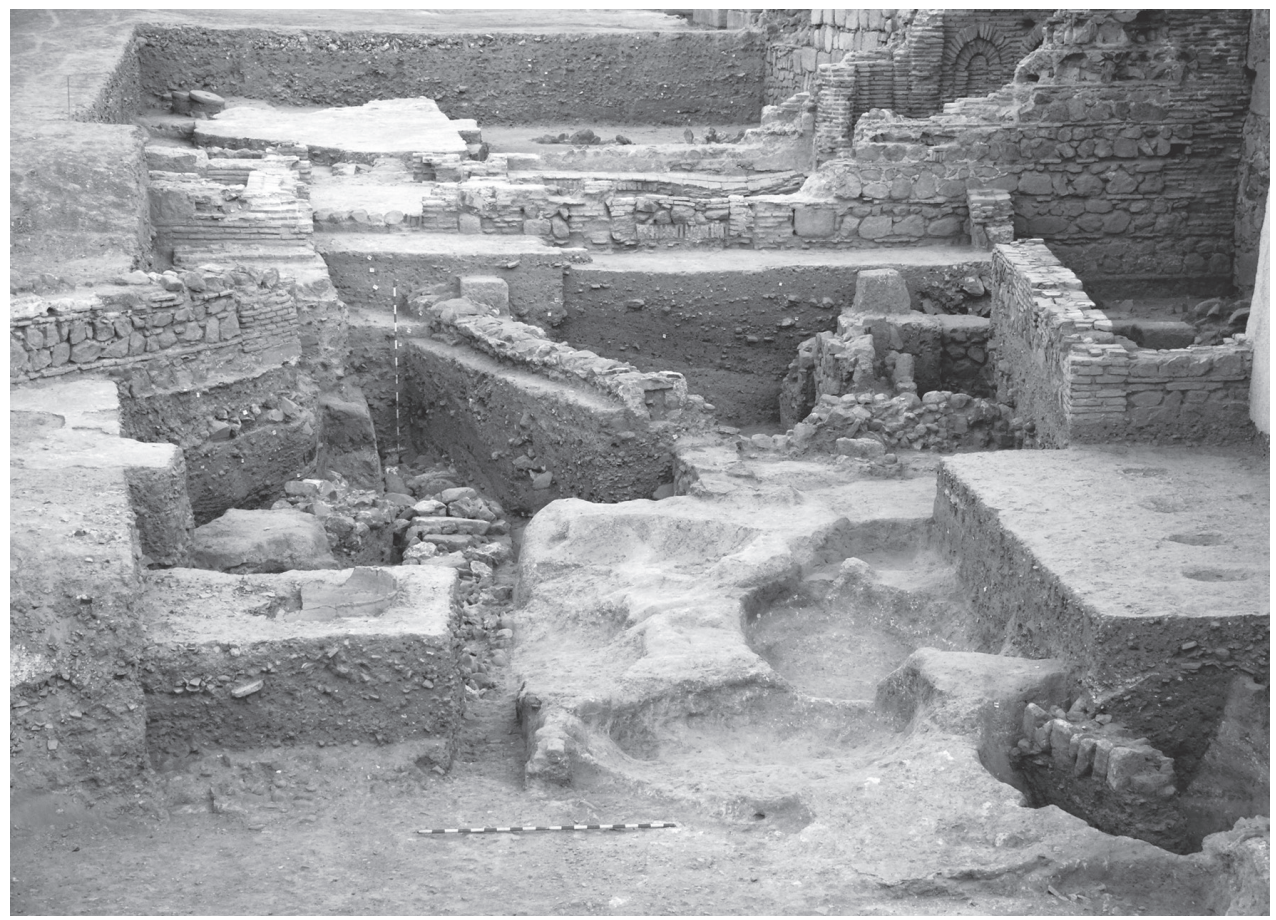

Fig. 2. Espacio donde se encuentran los basureros (Moraleda y Sánchez).

\section{Área 2}

En éste área fueron hallados varios testimonios numismáticos distribuidos en diferentes localizaciones. En primer lugar tenemos una serie de monedas ubicadas en los siguientes niveles deposicionales: UUEE 2025 (n. ${ }^{\circ}$ 2, s. III, Galieno) y 2036 (n. ${ }^{\circ}$ 34, s. XVI, Reyes Católicos ${ }^{1}$ y n. ${ }^{\circ}$ 43, s. XVII, Felipe IV). Estos niveles se encuentran cubriendo los restos de un pavimento de guijarros (UE 2038) corres-

\footnotetext{
${ }^{1}$ El comentario por épocas históricas de las piezas de los Reyes Católicos es complejo, al tratarse de emisiones encuadradas entre el final de la Edad Media y principios de la Edad Moderna. Es importante señalar que las piezas acuñadas en vellón y en plata se acuñaron hasta el año 1566. En las fichas del catálogo se especifica la datación de cada pieza con mayor precisión.
} 
pondiente a la ocupación de esta zona por la congregación Agustina, lo cual nos indica que son fruto de un relleno en época contemporánea, momento en el cual ya se había abandonado el recinto. Esta serie de niveles de rellenos llegarán hasta el momento en que este espacio se utiliza como huerto.

Otro grupo de monedas se han localizado en las UUEE: 2045 (n. ${ }^{\circ} \mathbf{7}$, pieza fechada en el s. IV d.C.); 2051 (n. ${ }^{\circ}$ 22, ss. XIV-XV, Enrique III y n. ${ }^{\circ}$ 27, s. XV, Enrique IV); 2053 (n. 23, ss. XIV-XV, Enrique III) y 2057 (n. ${ }^{\circ} 29$, s. XV, Enrique IV). Todos estos niveles deposicionales se hallaban bajo el solado de canto de río implantado por los Agustinos (U.E. 2038) y han sido interpretados como rellenos que fueron cubriendo los restos de las estructuras Medievales.

En los siguientes niveles también fueron halladas varias monedas: UUEE 2064 (n. ${ }^{\circ}$ 39, ss. XVI-XVII, Felipe III); 2065 (n. ${ }^{\circ}$ 38, ss. XVI-XVII, Felipe III) y 2087 (n. ${ }^{\circ}$ 37, ss. XVI-XVII, Felipe III). Estos niveles se interpretan como las primeras capas de tierra que cubrieron los restos de la nave y el ábside de la capilla (Fig. 3). Lo que puede confirmar que durante las primeras décadas del siglo XVII se abandona este espacio, el cual no se vuelve a usar hasta que se implanta la Congregación Agustina.

Por último, fueron también documentadas otras monedas en las UUEE 2102 (n. ${ }^{\circ}$ 8, ss. IV-V d.C., Honorio) y 2134 (n. ${ }^{\circ}$ 9, s. XII, Alfonso I²). Las cuales se encontraban en unos niveles de relleno de época altomedieval y anteriores a la instalación de una serie de infraestructuras de saneamiento de cronología medieval.

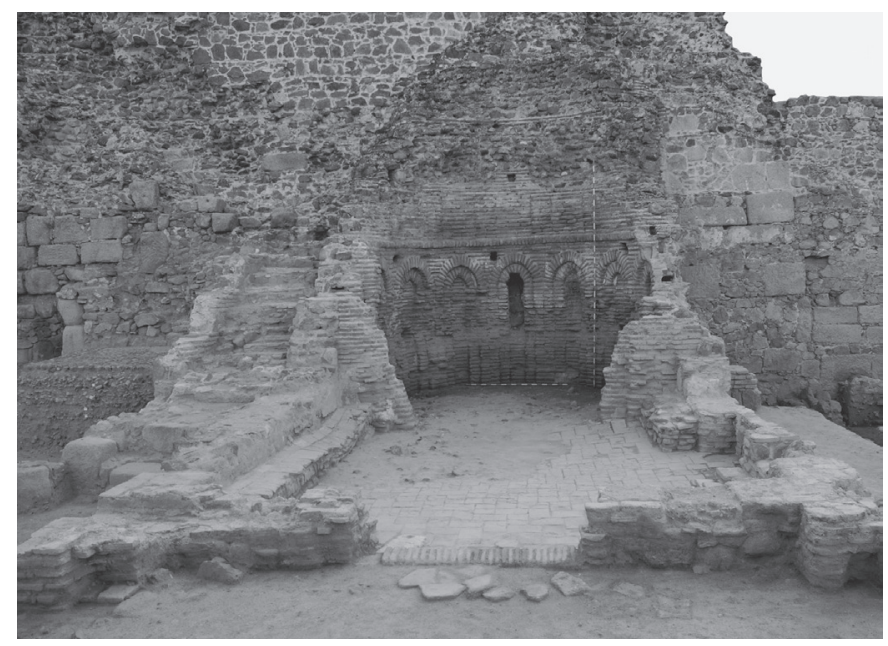

Fig. 3. Restos de la capilla (Moraleda y Sánchez).

2 Nos referimos a Alfonso I de Aragón, quien acuñó moneda en Castilla tras contraer matrimonio con Doña Urraca en 1109. A pesar de que su matrimonio se rompe en 1114, Alfonso I seguirá gobernando en Castilla hasta mediados de los años 20. 
Área 3

En este espacio se han hallado un total de 15 monedas. En general, el contexto en el que se han localizado estas monedas corresponde en su mayoría a niveles de relleno que colmatan varios hoyos-basureros registrados en este sector ${ }^{3}$ (Fig. 2). Dichos niveles corresponden con las siguientes UUEE: 3048 (n. ${ }^{\circ}$ 15, s. XIII, Alfonso X); 3084 (n. ${ }^{\circ}$ 11, s. XII, Alfonso I); 3095 (n. ${ }^{\circ}$ 33, ss. XV-XVI, Reyes Católicos); 3128 (n. ${ }^{\circ}$ 32, ss. XV-XVI, Reyes Católicos); 3133 (n. ${ }^{\circ}$ 4, s. II d.C.); 3136 (n. ${ }^{\circ}$ 19, s. XIV, Alfonso XI); 3138 (n. ${ }^{\circ}$ 20, s. XIV, Enrique II); 3151 (n. ${ }^{\circ}$ 5, s. IV d.C., Constantino I); 3156 (n..$^{\circ}$ 14, s. XIII, Alfonso X); 3162 (n. ${ }^{\circ}$ 24, s. XV, Juan II); 3185 (n. ${ }^{\circ}$ 26, s. XV, Enrique IV); 3186 (n..$^{\circ} 12$, s. XIII, Alfonso X y n. ${ }^{\circ} 13$, s. XIII, Alfonso X); 3191 (n. ${ }^{\circ} 16$, ss. XIII-XIV, Fernando IV) y 3273 (n. ${ }^{\circ} 49$, Romana- frustra).

\section{Área 4}

En éste área se han recuperado un total de once monedas. Los niveles donde fueron halladas las monedas corresponden con las UUEE: 4033 (n. ${ }^{\circ} 1$ S. II d.C.); 4036 (n. ${ }^{\circ}$ 31, ss. XV-XVI, Reyes Católicos); 4072 (n. ${ }^{\circ}$ 30, ss. XV-XVI, Reyes Católicos); 4125 (n. ${ }^{\circ}$ 25, s. XV, Enrique IV); 4137 (n. ${ }^{\circ}$ 10, s. XII, Alfonso I y n. ${ }^{\circ}$ 17, ss. XIII-XIV, Fernando IV); 4142 (n. ${ }^{\circ}$ 45, s. XVII, Felipe IV); 4154 (n. ${ }^{\circ}$ 40, s. XVII, Felipe IV y n. $^{\circ} 41$, s. XVII, Felipe IV); 4179 (n. ${ }^{\circ}$ 42, s. XVII, Felipe IV).

Estas monedas se encuentran en estratos deposicionales, siendo éstos en su gran mayoría niveles de relleno de todo este espacio una vez que se abandona y se derrumban parte de los elementos constructivos, ocurriendo esto a principios del siglo XVI. Estos rellenos se producirán también con la aportación de tierras durante la ocupación de todo este espacio por los padres agustinos.

\section{CONCLUSIONES}

Una vez realizado el estudio numismático hay que señalar que para aportar algún dato sobre la circulación monetaria en este lugar y la incidencia que tiene la aparición de estas piezas en la Alcazaba de Talavera, debemos circunscribirnos a la posible correlación entre las monedas y su contexto histórico y arqueológico. En este sentido, podemos establecer las siguientes valoraciones:

En líneas generales, los niveles documentados en las áreas 1, 2 y 4; puestos en relación con el registro numismático permite proponer, a modo de hipótesis,

\footnotetext{
${ }^{3}$ Hoyos, que tienen un origen en época Tardoantigua y cuya funcionalidad sería de extracción de arcilla y elaboración de adobes. Los cuales posteriormente en época medieval pierden su uso original, pasando a ser basureros colmatados por niveles medievales. Con posterioridad estos niveles quedarán amortizados por estructurales pertenecientes a remodelaciones de época Cristiana.
} 
que se tratan de niveles de relleno que colmatan todo este espacio durante época moderna una vez que las estructuras del interior del recinto se encuentran en avanzado estado de ruina. Se trata también de niveles con aportaciones de la Congregación Agustina, cuando el recinto interior de la Alcazaba comenzó a ser reutilizado como Huerto.

En el área 3, el contexto del material numismático se encuentra en relación con niveles de relleno de varios hoyos-basureros de cronología medieval y que quedarán amortizados por las reformas estructurales llevadas a cabo durante la época del alcázar cristiano.

Así mismo, los hallazgos numismáticos que pertenecen a época antigua y tardoantigua, se encuentran descontextualizados, aunque confirman la circulación monetaria durante estos periodos en la urbe, lo cual ha sido constatado en otras intervenciones realizadas en la ciudad (Urbina, 1995: 18-38) y en zonas circundantes: La Alcoba (De la Llave y Moraleda, 2009: 9-24) o en El Saucedo (Cabello Briones, 2008).

En lo que concierne a las cecas, de época antigua y tardoantigua, tan sólo tenemos constancia de la ceca en tres monedas, predominando la ceca Roma. Mayor información aporta el período medieval, donde predominan las cecas de algunas grandes urbes interiores de la península como Toledo, Cuenca y Burgos. Por lo que respecta a la época moderno-contemporánea, predominan las cecas de Toledo, Cuenca y Segovia, teniendo también menor presencia las cecas de Burgos, Sevilla, Granada y Madrid.

\section{CATÁLOGO}

Alto Imperio Romano

\section{N. ${ }^{\circ} 1$}

Identificación: TA/09/ALCAZABA/AREA4/SUBAREAI/4033

Gobierno/Rey: Alto Imperio romano.

Datación: Siglo II d.C.

Anv. Busto a la d.

Rev. Divinidad sedente a la i.

Valor: Sextercio; Ceca: No identificable.

Peso: 23,1 grs.; Módulo: 30 mm.; Metal: AE.

Obs. Oxidaciones en anverso y reverso. Elevado desgaste.

Bajo Imperio Romano 


\section{N. ${ }^{\circ} 2$}

Identificación: TA/09/ALCAZABA/AREA2/2025

Gobierno/Rey: Galieno.

Datación: 254 d.C.

Anv. GALLIENVS AVG. Busto radiado a la d.

Rev. PAX AVG. Paz estante a la i.

Valor: Antoniniano; Ceca: Roma.

Peso: 3,1 grs.; Módulo: 18 mm.; Metal: VE.

Obs. Oxidaciones en reverso.

Ref. RIC V 256.

\section{N. ${ }^{\circ} 3$}

Identificación: TA/2010/ALCAZABA/SUPERFICIE

Gobierno/Rey: Galieno.

Datación: 253-268 d.C.

Anv. GAL [LIENVS AVG]. Busto radiado a la d.

Rev. [---].

Valor: Antoniniano; Ceca:--.

Peso: 2,2 grs.; Módulo: 19 mm.; Metal: VE.

Obs. Fuertes oxidaciones en anv. y rev., en proceso de mineralización.

\section{N. ${ }^{\circ} 4$}

Identificación: TA/2010/ALCAZABA/AREA3/3133

Gobierno/Rey: Imperio Romano.

Datación: Siglo II d.C.

Anv. [---]. Busto radiado a la d.

Rev. [---].

Valor: Antoniniano; Ceca: --.

Peso: 1,5 grs.; Módulo: 17 mm.; Metal: VE.

Obs. Fuertes oxidaciones en anv. y rev., en proceso de mineralización. 


\section{N. ${ }^{\circ} 5$}

Identificación: TA/2010/ALCAZABA/AREA3/3151

Gobierno/Rey: Constantino I.

Datación: 318-319 d.C.

Anv. [IMP CONSTA] NTINVS [PF AVG]. Busto, a la d., con casco y coraza.

Rev. [VICTORIAE LAETAE PRINC PERP]. Dos victorias, portando un escudo con la inscripción [---] sobre un altar.

Valor: Follis?; Ceca: --.

Peso: 2,1 grs.; Módulo: $11 \mathrm{~mm}$.; Metal: AE.

Obs. Oxidaciones y principios de mineralización en anv. y rev.

N. ${ }^{\circ} 6$

Identificación: TA/09/ALCAZABA/AREA1/1023

Gobierno/Rey: Teodosio I.

Datación: 379-395 d.C.

Anv. DN THEODOSIVS PF AVG. Busto diademado a la d.

Rev. GLORIA ROMANORUM.

Valor: Maiorina; Ceca: --.

Peso: 5,3 grs.; Módulo: 18 mm.; Metal: AE.

Obs. Oxidaciones en anv. y rev.

\section{N. ${ }^{\circ} 7$}

Identificación: TA/09/ALCAZABA/AREA2/2045

Gobierno/Rey: Bajo Imperio Romano.

Datación: Siglo IV d.C.

Anv. [---]. Busto diademado, a la d.

Rev. [---].

Valor: Maiorina; Ceca:--.

Peso: 4,6 grs.; Módulo: 24 mm.; Metal: AE.

Obs. Oxidaciones en anv. y rev. muy acusadas, síntomas de mineralización. 


\section{N. ${ }^{\circ} 8$}

Identificación: TA/2010/ALCAZABA/AREA2/SUBAREAI/2102

Gobierno/Rey: Honorio.

Datación: 393-423 d.C.

Anv. DN [HONO]RIVS PF AVG. Busto diademado a la d.

Rev. GLORIA ROMANORUM. Honorio, estante, de frente, cabeza a la dcha., portando estandarte y globo.

Valor: Maiorina; Ceca: Nicomedia, $3^{\underline{a}}$ oficina.

Exergo: SMNT.

Peso: 4,4 grs.; Módulo: 22 mm.; Metal: AE/2.

Obs. Oxidaciones en anverso y reverso, desgaste al uso, pérdida de pátina.

Ref. Sear 4252; C. 20; RIC 46 c.

Edad Media

\section{N. ${ }^{\circ} 9$}

Identificación: TA/2010/ALCAZABA/AREA2/2134

Gobierno/Rey: Alfonso I.

Datación: 1109-1126.

Anv. ANFVS REX, Busto a la i.

Rev. TOLETA, Estrellas en primer y cuarto cuartel.

Valor: Dinero; Ceca: Toledo.

Peso: 0,7 grs.; Módulo: 17 mm.; Metal: VE.

Obs. Oxidaciones y principios de mineralización en anv. y rev.

Ref. Cayón, 920; Álvarez Burgos, 24.

N. ${ }^{\circ} 10$

Identificación: TA/2010/ALCAZABA/AREA4/SUBAREA I/4137

Gobierno/Rey: Alfonso I.

Datación: 1109-1126.

Anv. ANFVS REX, Busto a la i. 
Rev. TOLETA, Estrellas en segundo y tercer cuartel

Valor: Dinero; Ceca: Toledo.

Peso: 0,6 grs.; Módulo: 17 mm.; Metal: VE.

Obs. Desgaste al uso.

Ref. Cayón, 919; Álvarez Burgos, 25.

N. ${ }^{\circ} 11$

Identificación: TA/2010/ALCAZABA/AREA3/3084

Gobierno/Rey: Alfonso I.

Datación: 1109-1126.

Anv. ANFVS REX, Busto a la i.

Rev. TOLETA, Estrellas en primer y cuarto cuartel

Valor: Dinero; Ceca: Toledo.

Peso: 0,7 grs.; Módulo: 17 mm.; Metal: VE.

Obs. Desgaste al uso.

Ref. Cayón, 920; Álvarez Burgos, 24.

N. ${ }^{\circ} 12$

Identificación: TA/2010/ALCAZABA/AREA3/3186

Gobierno/Rey: Alfonso X.

Datación: 1252-1284.

Anv. ALFONSUS REX CASTELLE ET LEGIONIS.

Rev. Anepígrafa, cuartelado, $1^{\circ}$ y $4^{\circ}$ castillo, $2^{\circ}$ y $3^{\circ}$ león rampante a la i.

Valor: Dinero blanco de la guerra; Ceca: --.

Peso: 0,4 grs.; Módulo: 17 mm.; Metal: VE.

Obs. Se encuentra partida por la mitad.

N. ${ }^{\circ} 13$

Identificación: TA/2010/ALCAZABA/AREA3/3186

Gobierno/Rey: Alfonso X. 
Datación: 1252-1284.

Anv. ALFONSUS REX CASTELLE ET LEGIONIS.

Rev. Anepígrafa, cuartelado, $1^{\circ}$ y $4^{\circ}$ castillo, $2^{\circ}$ y $3^{\circ}$ león rampante a la i.

Valor: Dinero blanco de la guerra; Ceca: --.

Peso: 0,7 grs.; Módulo: 17 mm.; Metal: VE.

N. ${ }^{\circ} 14$

Identificación: TA/09/ALCAZABA/AREA3/3156

Gobierno/Rey: Alfonso X.

Datación: 1252-1284.

Anv. CASTELLE. Castillo.

Rev. LEGIONIS. León a la i.

Valor: Obolo; Ceca: Sin ceca.

Peso: 0,4 grs.; Módulo: 14 mm.; Metal: VE.

Ref. Cayón, 1122; Álvarez Burgos, 280.

N. ${ }^{\circ} 15$

Identificación: TA/09/ALCAZABA/AREA3/3048

Gobierno/Rey: Alfonso X.

Datación: 1252-1284.

Anv. CASTELLE. Castillo.

Rev. LEGIONIS. León a la i.

Valor: Obolo; Ceca: Sin marca.

Peso: 0,4 grs.; Módulo: 14 mm.; Metal: VE.

Ref. Cayón, 1122; Álvarez Burgos, 280.

N. ${ }^{\circ} 16$

Identificación: TA/2010/ALCAZABA/AREA3/3191

Gobierno/Rey: Fernando IV.

Datación: 1295-1312. 
Anv. F. REX CASTELLE. Castillo.

Rev. ET LEGIONIS. León a la i.

Valor: Pepión; Ceca: Sin marca.

Peso: 0,7 grs.; Módulo: 20 mm.; Metal: VE.

Ref. Cayón, 1167.

N. ${ }^{\circ} 17$

Identificación: TA/2010/ALCAZABA/AREA3/4137

Gobierno/Rey: Fernando IV.

Datación: 1295-1312.

Anv. F. REX CASTELLE. Castillo.

Rev. ET LEGIONIS. León a la i.

Valor: Pepión; Ceca: Cuenca (Cuenco).

Peso: 0,8 grs.; Módulo: 19 mm.; Metal: VE.

Ref. Cayón, 1161; Álvarez Burgos, 322.

N. ${ }^{\circ} 18$

Identificación: TA/09/ALCAZABA/AREA1/1023

Gobierno/Rey: Musulmán-Hispanomusulmán.

Datación: Siglos X-XIV d.C.

Anv. ¿Escritura cúfica?.

Rev. ¿Escritura cúfica?.

Valor: Felus; Ceca:--.

Peso: 0,6 grs.; Módulo: 14 mm.; Metal: AE.

Obs. Oxidaciones en anverso y reverso, síntomas de mineralización, rotura.

N. ${ }^{\circ} 19$

Identificación: TA/2010/ALCAZABA/AREA3/3136

Gobierno/Rey: Alfonso XI.

Datación: 1312-1350. 
Anv. ALF REX CASTELLE, Castillo.

Rev. ET LEGIONIS, León rampante a la i.

Valor: Noven; Ceca: Toledo.

Peso: 0,6 grs.; Módulo: 19 mm.; Metal: VE.

Ref. Cayón, 1190; Álvarez Burgos, 359.1.

N. ${ }^{\circ} 20$

Identificación: TA/2010/ALCAZABA/AREA3/3138

Gobierno/Rey: Enrique II.

Datación: 1369-1379.

Anv. ENRICUS REX LEGIONIS, Busto coronado a la i. Rosa detrás del busto.

Rev. ENRICUS REX CASTELLE, cruz, en los cuarteles E-N-R-I.

Valor: Cruzado; Ceca: Burgos.

Peso: 1,3 grs.; Módulo: 17 mm.; Metal: VE.

Obs. Presenta pequeña grieta u orificio.

Ref. Cayón, 1280.

N. ${ }^{\circ} 21$

Identificación: TA/2010/ALCAZABA/AREA4/SUBAREA2

Gobierno/Rey: Enrique II.

Datación: 1369-1379.

Anv. ENRICUS REX. Busto coronado a la i.

Rev. CASTELLE ET LEGIONIS. Castillo.

Valor: Cornado; Ceca: Burgos.

Peso: 1,2 grs.; Módulo: 19 mm.; Metal: VE.

Obs. Desgaste al uso.

Ref. Cayón, 1301; Álvarez Burgos, 486. 


\section{N. ${ }^{\circ} 22$}

Identificación: TA/2010/ALCAZABA/AREA2/SUBAREAI/2051

Gobierno/Rey: Enrique III.

Datación: 1390-1406.

Anv. ENRICUS DEI GRACIA. Castillo.

Rev. ENRICUS DEI GRACIA. León coronado a la i.

Valor: Blanca de 2 cornados; Ceca: Cuenca (Cuenco).

Peso: 2,2 grs.; Módulo: 23 mm.; Metal: VE.

Obs. Oxidaciones en anverso y reverso. En proceso de mineralización.

Ref. Cayón, 1412; Álvarez Burgos, 600.

\section{N. ${ }^{\circ} 23$}

Identificación: TA/2010/ALCAZABA/AREA2/2053

Gobierno/Rey: Enrique III.

Datación: 1390-1406.

Anv. ENRICVS REX CASTELLE. Castillo.

Rev. ENRICVS REX CASTELLE. León rampante a la i.

Valor: Blanca de 2 cornados; Ceca: Sevilla.

Peso: 1,7 grs.; Módulo: 24 mm.; Metal: VE.

Ref. Cayón, 1414; Álvarez Burgos, 609.2.

\section{N. ${ }^{\circ} 24$}

Identificación: TA/2010/ALCAZABA/AREA3/3162

Gobierno/Rey: Juan II.

Datación: 1430-1454.

Anv. IOHANES DEI GRACIA REX CA, Castillo.

Rev. IOHANES DEI GRACIA REX, León rampante a la i.

Valor: Blanca; Ceca: Toledo.

Peso: 2,0 grs.; Módulo: 23 mm.; Metal: VE.

Ref. Cayón, 1439; Álvarez Burgos, 629. 


\section{N. ${ }^{\circ} 25$}

Identificación: TA/09/ALCAZABA/AREA4/SUBAREAI/4125

Gobierno/Rey: Enrique IV.

Datación: 1454-1474.

Anv. ENRICUS DEI GRACIA REX.

Rev. ENRICUS DEI GRACIA REX.

Valor: Blanca; Ceca: Burgos.

Peso: 2 grs.; Módulo: 25 mm.; Metal: VE.

Obs. Cierto desgaste al uso.

Ref. Álvarez Burgos, 828.

\section{N. ${ }^{\circ} 26$}

Identificación: TA/2010/ALCAZABA/AREA3/3185

Gobierno/Rey: Enrique IV.

Datación: 1454-1474.

Anv. ENRICUS DEI GRACIA REX. Castillo.

Rev. ENRICUS DEI GRACIA REX. León rampante, a la i.

Valor: Blanca; Ceca: Burgos?.

Peso: 2,7 grs.; Módulo: 24 mm.; Metal: VE.

Obs. Pequeños síntomas de mineralización en rev.

Ref. Álvarez Burgos, 828.

\section{N. ${ }^{\circ} 27$}

Identificación: TA/2010/ALCAZABA/AREA2/SUBAREAI/2051

Gobierno/Rey: Enrique IV.

Datación: 1454-1474.

Anv. ENRICUS DEI GRACIA. Castillo.

Rev. XPS VINCIT XPS REG. León coronado a la i.

Valor: Blanca de rombo; Ceca: Toledo.

Peso: 1,8 grs.; Módulo: 20 mm.; Metal: VE. 
Obs. Oxidaciones en anverso y reverso. En proceso de mineralización.

Ref. Álvarez Burgos, 835.

\section{N. ${ }^{\circ} 28$}

Identificación: TA/2010/ALCAZABA/SUPERFÍCIE

Gobierno/Rey: Enrique IV.

Datación: 1471-1474.

Anv. ENRICUS DEI GRACIA. Castillo.

Rev. XPS VINCIT XPS REG. León coronado a la i.

Valor: Blanca de rombo; Ceca: Segovia.

Peso: 1,3 grs.; Módulo: 20 mm.; Metal: VE.

Obs. Oxidaciones en anv. y rev., en proceso de mineralización.

Ref. Álvarez Burgos, 833.

\section{N. ${ }^{\circ} 29$}

Identificación: TA/2010/ALCAZABA/AREA2/CAPILLA/2057

Gobierno/Rey: Enrique IV.

Datación: 1471-1474.

Anv. ENRICVS CUARTVS DEI. Castillo.

Rev. XPS VINCIT XPS. León rampante a la i.

Valor: Blanca de rombo; Ceca: Burgos.

Peso: 1 grs.; Módulo: 20 mm.; Metal: VE.

Obs. Oxidaciones en anv. y rev.

Ref. Álvarez Burgos, 828.

Final Edad Media - Principios Edad Moderna

\section{N. ${ }^{\circ} 30$}

Identificación: TA/09/ALCAZABA/AREA4/SUBAREAI/4072

Gobierno/Rey: Reyes Católicos.

Datación: 1520-1566. 
Anv. FERNANDVS ET ELISABET. Castillo, alrededor orla de 6 lóbulos.

Rev. REX ET REGINA CAST LEGION. León, a la i., alrededor orla de 6 lóbulos.

Valor: Un cuarto; Ceca: --.

Peso: 5.4 grs.; Módulo: 30 mm.; Metal: AE.

Obs. Oxidaciones en anverso y reverso.

N. ${ }^{\circ} 31$

Identificación: TA/09/ALCAZABA/AREA4/SUBAREAI/4036

Gobierno/Rey: Reyes Católicos.

Datación: 1497-1566.

Anv. FERNANDVS ET ELISABET. F coronada, en el campo, a la d., punto.

Rev. REX ET REGINA CAST LEGION. Y coronada.

Valor: Blanca; Ceca: Segovia?.

Peso: 0,8 grs.; Módulo: $17 \mathrm{~mm}$.; Metal: VE.

Obs. Oxidaciones en anverso y reverso.

N. ${ }^{\circ} 32$

Identificación: TA/09/ALCAZABA/AREA3/3128

Gobierno/Rey: Reyes Católicos.

Datación: 1497-1566.

Anv. FERNANDVS ET ELISABET. F coronada.

Rev. REX ET REGINA CAST LEGION. Y coronada.

Valor: Blanca; Ceca: Toledo.

Peso: 0,9 grs.; Módulo: 19 mm.; Metal: VE.

Obs. Oxidaciones en anverso y reverso. Elevado desgaste.

N. ${ }^{\circ} 33$

Identificación: TA/09/ALCAZABA/AREA3/3095

Gobierno/Rey: Reyes Católicos.

Datación: 1497-1566. 
Anv. FERNANDVS ET ELISABET. F coronada, en el campo, a la d., punto.

Rev. REX ET REGINA CAST LEGION. Y coronada.

Valor: Blanca; Ceca: --.

Peso: 0,9 grs.; Módulo: 20 mm.; Metal: VE.

Obs. Deformada y agrietada.

\section{N. ${ }^{\circ} 34$}

Identificación: TA/09/ALCAZABA/AEA2/2036

Gobierno/Rey: Reyes Católicos.

Datación: 1520-1566.

Anv. Ilegible. Castillo.

Rev. Ilegible. León, a la i.

Valor: Ochavo.; Ceca: --.

Peso: 3,1 grs.; Módulo: 23 mm.; Metal: AE.

Obs. Oxidaciones en anverso y reverso, síntomas de mineralización, rotura.

Edad Moderna

\section{N. ${ }^{\circ} 35$}

Identificación: TA/2010/ALCAZABA/AREA2/INT.ABSIDEBAJOSOLADO.

Gobierno/Rey: Felipe II.

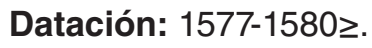

Anv. Castillo.

Rev. León a la d.

Valor: 2 maravedís; Ceca: Granada.

Peso: 2,9 grs.; Módulo: 21 mm.; Metal: AE.

Obs. Desgaste al uso.

Ref. C-96. 


\section{N. ${ }^{\circ} 36$}

Identificación: TA/2007/INTERIOR MURO1

Gobierno/Rey: Felipe II.

Datación: 1566-1582 aprox.

Anv. PHS. Encima corona Real.

Rev. Castillo.

Valor: Blanca; Ceca: Cuenca.

Peso: 1 grs.; Módulo: 16 mm.; Metal: AE.

Obs. Mineralizaciones en anv., desgaste al uso.

Ref. López de la Fuente, C-17.

N. ${ }^{\circ} 37$

Identificación: TA/2010/ALCAZABA/AREA2/2087

Gobierno/Rey: Felipe III.

Datación: [ilegible] 1598-1602.

Anv. PHILIPPUS III D.G. Castillo.

Rev. HISPANIARUM REX. León rampante a la i.

Valor: 4 maravedís; Ceca: Madrid (MD).

Peso: 1,5 grs.; Módulo: 14 mm.; Metal: AE.

Obs. Oxidaciones en anv. y rev.

\section{N. ${ }^{\circ} 38$}

Identificación: TA/2010/ALCAZABA/AREA2/2065

Gobierno/Rey: Felipe III.

Datación: 1605.

Anv. PHILIPPUS III D.G. Castillo.

Rev. HISPANIARUM REX. León rampante a la i.

Valor: 2 maravedís; Ceca: Segovia.

Peso: 1,9 grs.; Módulo: 16 mm.; Metal: AE. 
Obs. Oxidación y mineralización en rev.

Ref. Cayón, 4016.

\section{N. ${ }^{\circ} 39$}

Identificación: TA/2010/ALCAZABA/AREA2/2064

Gobierno/Rey: Felipe III.

Datación: 1605.

Anv. PHILIPPUS III D.G. Castillo.

Rev. HISPANIARUM REX. León rampante a la i.

Valor: 2 maravedís; Ceca: Segovia.

Peso: 1,9 grs.; Módulo: 14 mm.; Metal: AE.

Obs. Ligeras oxidaciones en anv. y rev.

Ref. Cayón, 4016.

\section{N. ${ }^{\circ} 40$}

Identificación: TA/2010/ALCAZABA/AREA4/SUBAREAI/4154

Gobierno/Rey: Felipe IV.

Datación: 1624.

Anv. PHILIPPUS IIII. D.G. Castillo, dentro de escudo coronado.

Rev. HISPANIARUM REX. León rampante a la i, dentro de escudo coronado,

Valor: 8 maravedís; Ceca: Burgos.

Peso: 6,2 grs.; Módulo: 23 mm.; Metal: AE.

Obs. Oxidaciones en anv. y rev.

Ref. Cayón, 5043; López de la Fuente, B-4.

\section{N. ${ }^{\circ} 41$}

Identificación: TA/2010/ALCAZABA/AREA4/SUBAREAI/4154

Gobierno/Rey: Felipe IV.

Datación: 1621-1626.

Anv. PHILIPPUS IIII. D.G. Castillo, dentro de escudo coronado. 
Rev. HISPANIARUM REX. León rampante a la i, dentro de escudo coronado,

Valor: 8 maravedís; Ceca:--.

Peso: 6,2 grs.; Módulo: 24 mm.; Metal: AE.

Obs. Oxidaciones en anv. y rev.

N. ${ }^{\circ} 42$

Identificación: TA/2010/ALCAZABA/AREA4/SUBAREAI/4179

Gobierno/Rey: Felipe IV.

Datación: 1621-1626.

Anv. [PHILIPPUS IIII. D.G.] Castillo, dentro de escudo coronado.

Rev. [HISPANIARUM REX.] León rampante a la i, dentro de escudo coronado, Valor: 8 maravedís; Ceca:--.

Peso: 5,1 grs.; Módulo: 22 mm.; Metal: AE.

Obs. Oxidaciones en anv y rev, restos de mineralización y arañazos en anv.

\section{N. ${ }^{\circ} 43$}

Identificación: TA/09/ALCAZABA/AREA2/2036

Gobierno/Rey: Felipe IV.

Datación: 1641.

Anv. Resello año 1641, en su parte superior corona real.

Rev. Resellos de ocho maravedís, debajo acueducto.

Valor: 8 maravedís.; Ceca: --.

Peso: 7,4 grs.; Módulo: $21 \mathrm{~mm}$.; Metal: AE.

Obs. Oxidaciones en anverso y reverso, síntomas de mineralización.

\section{N. ${ }^{\circ} 44$}

Identificación: TA/2007/ALCAZABA/T-65

Gobierno/Rey: Felipe IV.

Datación: 1641.

Anv. Resello de VIII Maravedís, debajo MD. 
Rev. Resello año 1641 bajo corona real.

Valor: 8 Maravedís; Ceca: Madrid.

Peso: 6,6 grs.; Módulo: 21 mm.; Metal: AE.

Ref. Cayón, 5074.

N. ${ }^{\circ} 45$

Identificación: TA/2010/ALCAZABA/AREA4/SUBAREAI/4142

Gobierno/Rey: Felipe IV.

Datación: 1636-1642.

Anv. [---].

Rev. [---].

Valor: Resello 6 maravedís; Ceca:--.

Peso: 5,4 grs.; Módulo: 24 mm.; Metal: AE.

Obs. Oxidaciones en anv. y rev.

\section{N. ${ }^{\circ} 46$}

Identificación: TA/09/ALCAZABA/VANO BARBACANA JUNTO A T-1

Gobierno/Rey: Felipe IV.

Datación: 1651.

Anv. Resello de 8 maravedís.

Rev. Resello año 1651.

Valor: 8 maravedís; Ceca: --.

Peso: 6,4 grs.; Módulo: 28 mm.; Metal: AE.

N. ${ }^{\circ} 47$

Identificación: TA/2007/ALCAZABA/SUPERFÍCIE

Gobierno/Rey: Felipe IV.

Datación: 1663.

Anv. PHILIPPVS IIII D.G. Busto de Felipe IV a la d.

Rev. HISPANIARVM REX. Escudo Real. 
Valor: 16 maravedís; Ceca: Madrid (M-S).

Peso: 4,1 grs.; Módulo: 27 mm.; Metal: AE.

Obs. Ligeramente descentrada. Ensayador: Sebastián González de Castro.

Ref. López de la Fuente, E-640.

\section{N. ${ }^{\circ} 48$}

Identificación: TA/2010/ALCAZABA/SUPERFICIE

Gobierno/Rey: Carlos II.

Datación: 168[-].

Anv. [CARO]LU[S] II [D.G.]. Castillo, dentro de escudo coronado.

Rev. HISP[ANIARUM REX]. León rampante a la i., dentro de escudo coronado.

Valor: 2 maravedís; Ceca: Madrid (MD).

Peso: 6,0 grs.; Módulo: 20 mm.; Metal: AE.

\section{N. ${ }^{\circ} 49$}

Identificación: TA/2010/ALCAZABA/AREA3/SUBAREAI/3273

Gobierno/Rey: Frustra.

Datación: --.

Anv. --.

Rev. --.

Valor: --; Ceca:--.

Peso: 0,9 grs.; Módulo: 19 mm.; Metal: AE.

Obs. En proceso avanzado de mineralización. 


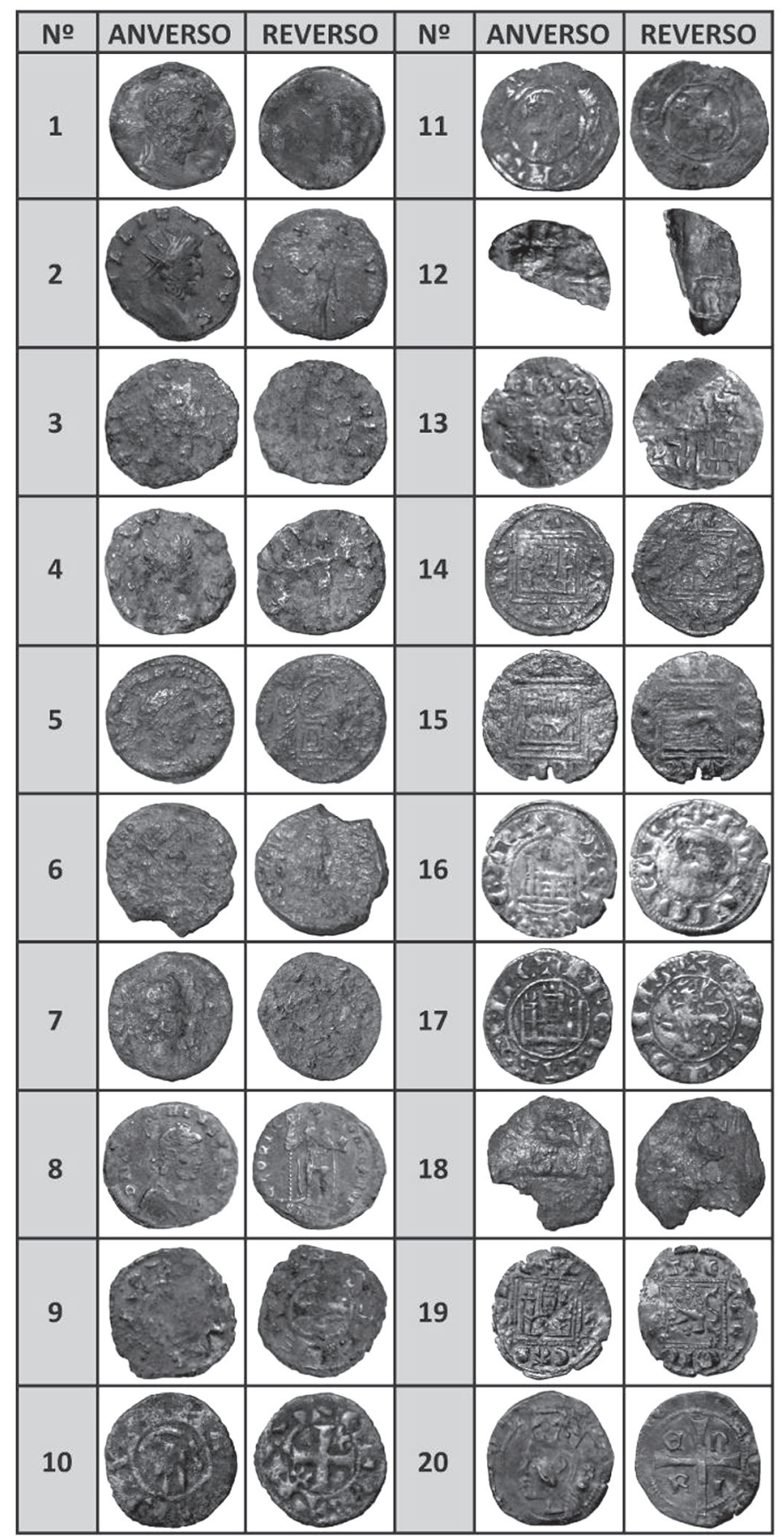

Tabla 1. Monedas del $n .{ }^{\circ} 1$ al 20 (Autores). 


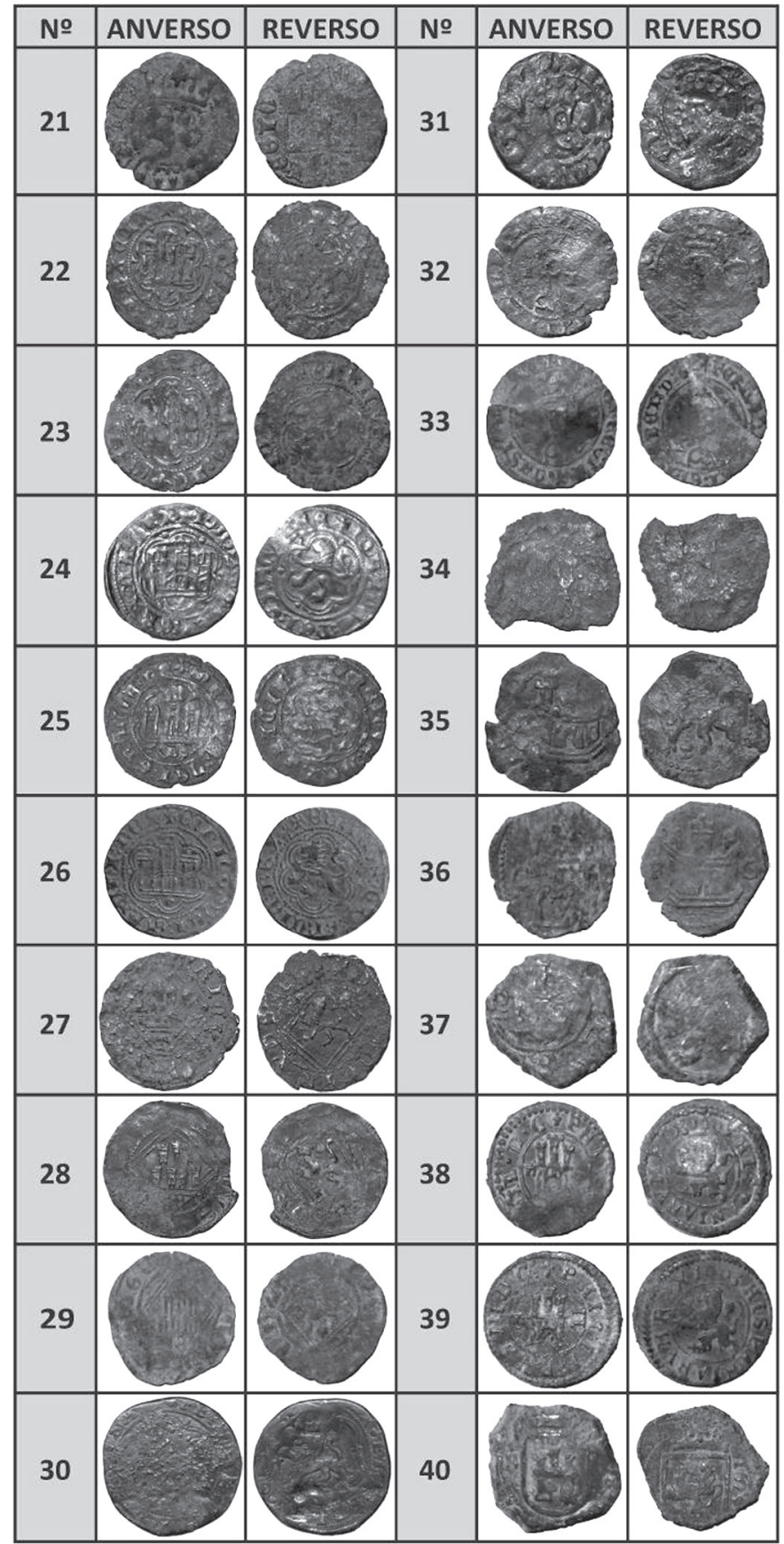

Tabla 2. Monedas del $n .{ }^{\circ} 21$ al 40 (Autores). 


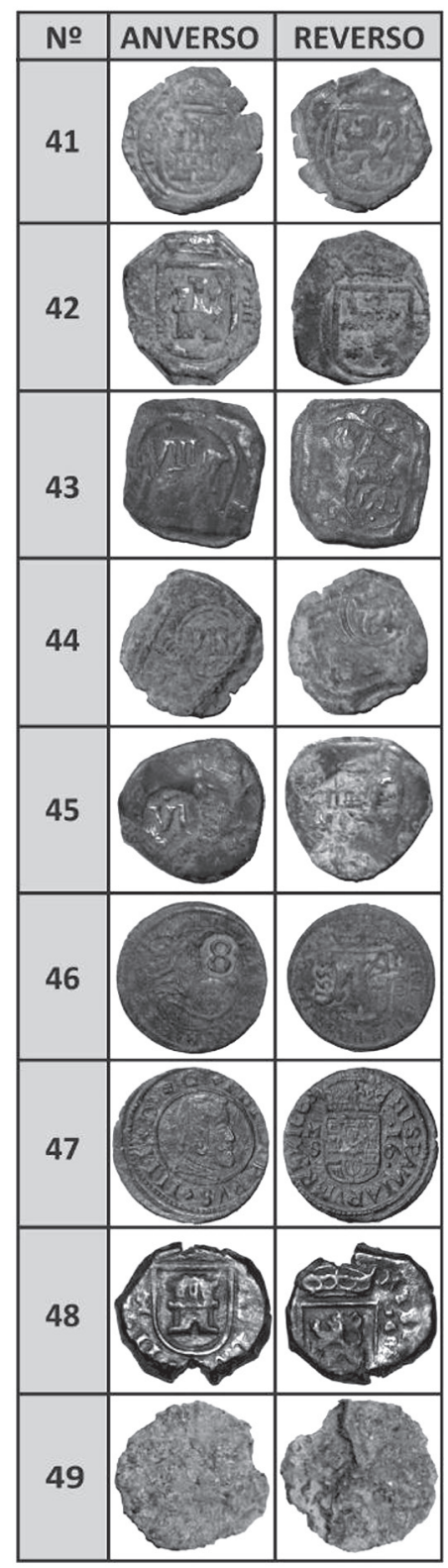

Tabla 3. Monedas del n. ${ }^{\circ} 41$ al 49 (Autores). 


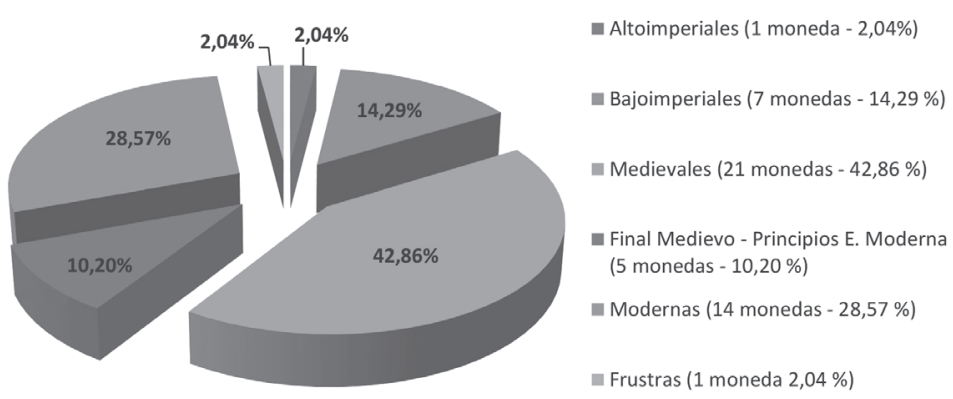

Gráfico 1. Porcentaje de monedas halladas por periodos (Autores).

\section{BIBLIOGRAFÍA}

ÁLVAREZ BURGOS, F. (1998): Catálogo de la moneda medieval Castellano-Leonesa siglos XI a XV, Madrid.

BRAÑA PASTOR, J. L. y ROMA VALDÉS A. (2010): El vellón Castellano del siglo XV.

CABELLO BRIONES, A. M. (2008): Moneda e Historia en Tierras de Talavera: Los hallazgos monetarios del yacimiento de El Saucedo (Talavera la Nueva. Toledo), Excmo. Ayuntamiento de Talavera de la Reina.

CANTO GARCÍA, A. y RODRíGUEZ CASANOVA, I. (2006): «Nuevos datos acerca de la inscripción califal atribuida al Castillo de Baños de la Encina (Jaén)», AyTM, 13.2, pp. 57-66.

CASILLAS ROLLÓN, A. (2012): «Medina del Campo 1497: Análisis de la reforma monetaria de los Reyes Católicos", Ab Initio, Número Extra, 2, pp. 57-89.

CAYÓN, A.; CAYÓN, C. y CAYÓN, J. (1998): Las monedas Españolas. Del Tremis al Euro, del 411 a nuestros días, Madrid, Juan Cayón (Ed.).

CENTENO YÁNEZ, J. (2006): Las monedas reselladas de Felipe III y Felipe IV (1603-1659). Estudio y catalogación, Córdoba.

COHEN, H. (1930): Description historique des monnaies frappés sous l'empire romain comunnement appe-lées médailles impériales, 8 vol., Leipzig.

DE FRANCISCO OLMOS, J. M. (1999): «La moneda castellana de los Reyes Católicos. Un documento económico y político", Revista General de Información y Documentación, Vol. 9, 1, pp. 85-115.

DE LA LLAVE MUÑOZ, S. y MORALEDA OLIVARES, A. (2008-2009): «Estudio de un conjunto de monedas halladas en La Alcoba (Talavera la Nueva, Toledo)», Cuaderna: Revista de estudios humanísticos de Talavera y su antigua tierra, 16 y 17, pp. 9-25.

FROCHOSO SÁNCHEZ, R. (2001): Los Feluses de Al-Ándalus, Córdoba.

LÉVI-PROVENÇAL, E. (1953): «La description de l’Espagne d'ahmad al-Râzî̀, Al-Ándalus, XVIII, pp. 51-108.

LÓPEZ DE LA FUENTE, J. L. (2011): Los maravedís de los Austrias (1516-1700): Tipos y variantes, Torredonjimeno.

LÓPEZ GAYARRE, P.A. (2011): Historia documental del urbanismo en Talavera (1450-1700), Excmo. Ayto. de Talavera de la Reina.

MARTÍNEZ LILLO, S. (1994): «Un ribat interior en la Marca Media. El caso de Talabíra», CUPUAM, 21, pp. 297-312. 
MARTÍNEZ LILLO, S. (1998): Arquitectura militar andalusí en la Marca Media. El caso de Talabíra, Excmo. Ayto. de Talavera de la Reina.

MARTíNEZ LILLO, S.; MORALEDA OLIVARES, A. y SÁNCHEZ SANZ, S. (2005): «El yacimiento arqueológico de Entretorres (Talavera de la Reina). Últimas portaciones del periodo andalusí’, en Actas del Congreso Espacios Fortificados en la Provincia de Toledo, pp. 117-154.

MORALEDA OLIVARES y SÁNCHEZ SANZ, S. (2009): Proyecto de intervención arqueológica en la Alcazaba de Talavera de la Reina (Toledo), Consejería de Cultura de la Junta de CastillaLa Mancha, Exp. 09.0530, Inédito.

MORALEDA OLIVARES y SÁNCHEZ SANZ, S. (2010): Proyecto de intervención arqueológica en la Alcazaba de Talavera de la Reina (Toledo), Consejería de Cultura de la Junta de CastillaLa Mancha, Exp. 09.0530-AP, Inédito.

MORALEDA OLIVARES, A. (2001): Proyecto de seguimiento y control arqueológico de la actuación de acondicionamiento del Huerto de San Agustín de Talavera de la Reina (Toledo), Consejería de Cultura de la Junta de Castilla-La Mancha, Inédito.

MORALEDA OLIVARES, A. (23-4-2002): «El hábitat de la antigua madina Talabíra, Historias de la Historia», La Voz de Talavera, pp. 20-21.

MORALEDA OLIVARES, A. y MAQUEDANO CARRASCO, B. (2002-2003): Intervención de los trabajos arqueológicos en la escuela taller de la Alcazaba, Consejería de Cultura de la Junta de Castilla-La Mancha, Inédito.

MORALEDA OLIVARES, A.; MARTÍNEZ LILLO, S. y SÁNCHEZ SANZ, S. (1999-2000): «Primeros resultados de los trabajos arqueológicos en Entretorres. Primer recinto de la muralla islámica de Talavera de la Reina", Cuaderna: Revista de estudios humanísticos de Talavera y su antigua tierra, 7-8, pp. 54-70.

MORALEDA OLIVARES, A.; MARTÍNEZ LILLO, S. y SÁNCHEZ SANZ, S. (2001): Memoria de excavación sector C/Charcón, Inédito.

MORALEDA OLIVARES, A.; MARTíNEZ LILLO, S. y SÁNCHEZ SANZ, S. (2005): Memoria de los trabajos arqueológicos desarrollados en los sectores Entretorres-Charcón, Consejería de Cultura de la Junta de Castilla-La Mancha, Exp. 05.0056, Inédito.

MORALEDA OLIVARES, A.; MARTÍNEZ LILLO, S. y SÁNCHEZ SANZ, S. (2006-2007): Proyecto de intervención arqueológica en la Alcazaba Omeya (Huerto de San Agustín) de Talavera de la Reina, Consejería de Cultura de la Junta de Castilla-La Mancha, Exp. 62.215, Inédito.

MORALEDA OLIVARES, A.; MARTíNEZ LILLO, S. y SÁNCHEZ SANZ, S. (2006): Memoria de los trabajos arqueológicos desarrollados en los sectores Entretorres-Charcón, Consejería de Cultura de la Junta de Castilla-La Mancha, Exp. 06.0695, Inédito.

MORALEDA OLIVARES, A.; MARTÍNEZ LILLO, S. y SÁNCHEZ SANZ, S. (2007): Memoria de los trabajos arqueológicos desarrollados los sectores Entretorres-Charcón, Consejería de Cultura de la Junta de Castilla-La Mancha, Exp. 07.0036, Inédito.

MORALEDA OLIVARES, A.; SÁNCHEZ SANZ, S. y MARTíNEZ LILLO, S. (2004): «EI Yacimiento de Entretorres (Talavera de la Reina)", en Investigaciones arqueológicas en Castilla-La Mancha (1996-2002), Consejería de Educación y Cultura, Junta de Comunidades de Castilla-La Mancha, pp. 409-421.

PACHECO JIMÉNEZ, C. (2008): «El espacio urbano de Medina Talabira (Talavera de la Reina). Una aproximación histórico-arqueológica», en Actas Al-Ándalus, país de ciudades, Diputación Provincial de Toledo, pp. 283-296.

SEAR, D.R. (1988): Roman Coins and their values, Londres, (1aㅡ edición 1981).

URBINA MARTÍNEZ, D. (1995): «El conjunto de monedas de la Ronda del Cañillo, 16», Cuaderna: Revista de estudios humanísticos de Talavera y su antigua Tierra, 2, pp. 18-38.

VILLA CONZÁLEZ, J. R. (1992): «Excavaciones arqueológicas en el Huerto de San Agustín», en Actas de las I Jornadas de arqueología de Talavera y sus tierras, Diputación Provincial de Toledo, pp. 371-392. 